\title{
Prévalence et facteurs de risque de la fièvre aphteuse dans le marché à bétail de Garoua-Boulai et dans la zone de transhumance du Lom et Djerem à l'Est du Cameroun
}

\author{
A. Sassa MEBANGA*, L. Salman BELOKO et Abdoulmoumini MAMOUDOU \\ Ecole des Sciences et de Médecine Vétérinaire - Département de Production Animale Université de \\ Ngaoundere, B.P. 268 Ngaoundere-Cameroun. \\ *Auteur correspondant ; E-mail: mebengasaaristide@gmail.com
}

\section{RESUME}

La fièvre aphteuse (FA) est une maladie transfrontalière qui affecte les ongulés domestiques et sauvages. Elle joue un rôle très important en économie du fait de sa morbidité élevée et de sa restriction du commerce international. En vue de déterminer la prévalence de la FA bovine et les facteurs de risque de transmission de la maladie au marché à bétail de Garoua-Boulaï et dans la zone de transhumance du département du Lom et Djerem à l'Est-Cameroun, une étude rétrospective et transversale a été menée de juin à décembre 2018. Au marché à bétail, l'étude a porté sur l'observation des signes cliniques caractéristiques sur des animaux suspects. Ainsi, 58 bovins atteints de FA ont été identifiés parmi les 614 bovins suspects, soit une prévalence clinique globale de 9,44\% (IC95\% : 7,13\% - 11,76\%). Les facteurs internes tels que le sexe n'ont pas influencé la prévalence de la FA. Dans la zone de transhumance, la prévalence rapportée au niveau individuel était de $26,77 \%$. Tous les éleveurs connaissent la FA et ont cité plus de 3 signes cliniques caractéristiques de la maladie. Le fait de considérer la FA comme une maladie mineure, la faible connaissance des sources, des réservoirs et du mode de contamination du virus aphteux par toute la chaine de valeur bovine, expliquent l'endémicité de la maladie dans ces zones.

(C) 2020 International Formulae Group. All rights reserved.

Mots clés : Prévalence, facteurs de risque, fièvre aphteuse bovine, Garoua-Boulai, Lom et Djerem.

\section{Prevalence and risk factors of foot-and-mouth disease at the Garoua-Boulai cattle market and in the transhumance area of the Lom and Djerem department in Eastern Cameroon}

\begin{abstract}
Foot-and-mouth disease (FMD) is a cross-border disease that affects domestic and wild ungulates. It plays a very important role in economics because of its high morbidity and its restriction of international trade. To determine the prevalence of FMD and the risk factors for transmission of the disease at the Garoua-Boulai cattle market and in the transhumance area of the Lom and Djerem division in Eastern Cameroon, a retrospective and transversal study was conducted from June to December 2018. At the cattle market, the study focused on the observation of characteristic clinical signs on suspect animals. Thus, 58 cattle with FMD were identified among the 614 suspected cattle, representing an overall clinical prevalence of 9, 44\% (IC95\%: 7, 13\% - 11, 76\%). Internal factors such gender did not influence prevalence of the FMD. In the transhumance area, the prevalence
\end{abstract}


reported at the individual level was 26,77\%. All breeders know about FMD and have cited more than 3 clinical signs characteristic of the disease. Considering FMD as a minor disease, poor knowledge of the sources, reservoirs and mode of contamination of FMD virus throughout the bovine value chain, explains the endemic nature of the disease in these areas.

(C) 2020 International Formulae Group. All rights reserved.

Keywords: Prevalence, risk factors, bovine foot-and-mouth disease, Garoua-Boulai, Lom and Djerem.

\section{INTRODUCTION}

Dans la région de l'Est du Cameroun, le système d'élevage des bovins est essentiellement extensif et se caractérise par la mobilité (transhumance) des animaux. Les mouvements des animaux se font d'une part de la région de l'Est vers la région du Centre qui dessert les régions du Sud, du Littoral et de l'Ouest; d'autre part les animaux sont exportés vers le Tchad, la République Centrafricaine, le Nigéria, la Guinée Equatoriale et le Gabon (Kuate, 2016).

La commercialisation des animaux et des denrées alimentaires d'origine animale connaissent une croissance considérable au fil des années. Le flux commercial a facilité la dissémination de certaines maladies dites transfrontalières telle que la fièvre aphteuse qui constitue un frein important pour le développement des filières animales de par sa forte morbidité atteignant parfois $100 \%$ et une mortalité quasi nulle chez les adultes mais élevée chez les jeunes (FAO, 2015).

La fièvre aphteuse (FA) est une maladie virale, hautement contagieuse, due à un virus appartenant au genre Aphthovirus de la famille des Picornaviridés. Elle touche tous les animaux à sabots fendus qu'ils soient domestiques ou sauvages (bovins, caprins, ovins, porcs et autres artiodactyles). Cette maladie revêt un caractère transfrontalier et entraîne des pertes économiques considérables notamment une réduction importante de la production de lait ou de viande dans les élevages infectés et des performances du commerce du bétail et de ses produits (Miguel et al., 2013). C'est une maladie à déclaration obligatoire. Elle est la première maladie pour laquelle l'Organisation Mondiale de la Santé Animale (OIE) a établi une liste officielle de pays et zones reconnues indemnes avec ou sans vaccination. Elle est endémique au Cameroun, mais n'est pas une zoonose. L'introduction de la maladie dans un troupeau se fait par de nouveaux animaux transportant le virus (dans la salive, le lait, la semence, etc.), l'utilisation d'enclos, de bâtiments ou de véhicules contaminés pour héberger et transporter des animaux sensibles, la présence de matériels contaminés tels que foin, aliments, eau, lait ou produits biologiques, le port de vêtements ou de chaussures contaminés ou l'utilisation d'équipements contaminés, la distribution à des animaux sensibles de viande, de produits d'origine animale, d'aliments crus ou mal cuits, contaminés par le virus ; la dissémination virale par des aérosols transportés par l'air à partir d'une exploitation contaminée. Son diagnostic est basé d'une part sur l'observation d'un ou des principaux signes cliniques et des lésions, et d'autre part sur les résultats des tests de laboratoire (ELISA et PCR). La prévention de la maladie passe par la biosécurité, l'abattage sanitaire et la vaccination. Il existe ainsi 07 sérotypes immunologiquement distincts qui circulent dans le monde dont 04 au Cameroun (Kuate, 2016). Les sérotypes identifiés au Cameroun par le Laboratoire National Vétérinaire (LANAVET) sont de type A, O, SAT1 et SAT2. Du fait de la multitude de sérotypes, le vaccin utilisé doit être spécifique à la souche virale circulant afin de mieux protéger les animaux exposés. Il n'existe aucun traitement spécifique contre la fièvre aphteuse, mais on peut aider à la guérison des plaies en appliquant des antiseptiques locaux et en administrant des antibiotiques de couverture pour empêcher les infections secondaires.

$\mathrm{Au}$ Cameroun où la maladie est endémique, très peu de cas de fièvre aphteuse sont rapportés en raison soit du système d'élevage qui est de type extensif, soit par 
manque de sensibilisation des populations locales, soit par manque de moyens et de volonté des acteurs. L'objectif de la présente étude est d'actualiser les données épidémiologiques en déterminant la prévalence et tous les facteurs de risque qui peuvent influencer l'état d'endémicité de la FA dans la région de l'Est afin d'éviter les pratiques à risque et d'envisager les stratégies appropriées de contrôle.

\section{MATERIEL ET METHODES \\ Zone d'étude}

L'étude s'est déroulée du $1^{\text {er }}$ juin au 30 décembre 2018 dans le département du Lom et Djerem dans la région de l'Est du Cameroun. Cette zone se situe entre le $5^{\circ} 14^{\prime} 11.645^{\prime \prime}$ de latitude Nord et le $13^{\circ} 54^{\prime} 51.836^{\prime \prime}$ de longitude Est. $\mathrm{Ce}$ département compte 08 arrondissements (Belabo, Bertoua $1^{\mathrm{er}}$, Bertoua $2^{\text {ème }}$, Bétaré-Oya, Diang, Garoua-Boulai Mandjou et Ngoura,) et couvre une superficie de $26345 \mathrm{~km}^{2}$ (Figure1). Le climat est de type tropical caractérisé par une grande saison de pluie d'avril à octobre avec un maximum de pluie en août, et une petite saison sèche de novembre à mars. La moyenne des précipitations annuelles est de $1500 \mathrm{~mm}$ et les températures moyennes oscillent entre $20^{\circ} \mathrm{C}$ et $26{ }^{\circ} \mathrm{C}$. Les activités principales sont l'agriculture, l'élevage, l'exploitation minière, la pêche, l'apiculture et le commerce. La zone est un point de transit entre le grand Nord et le grand Sud du Cameroun pendant la période de transhumance. Elle est également caractérisée par la porosité de la frontière avec la République Centrafricaine.

\section{Matériel animal - Echantillonnage}

Les recherches se sont déroulées au marché à bétail de Garoua-Boulai et dans la zone de transhumance du département du Lom et Djerem. Les races rencontrées sont essentiellement les zébus goudali, peulhs et bororos. Tous les animaux étaient issus d'élevages de type traditionnel. Les informations reçues des éleveurs et vendeurs d'animaux atteints cliniquement de FA ont permis d'identifier la zone de provenance des animaux.
Au marché à bétail de Garoua-Boulai, le plus grand marché frontalier du département du Lom et Djerem, une étude analytique basée sur l'observation des cas cliniques de FA a été réalisée. L'unité primaire était le troupeau dans le cadre de l'enquête épidémiologique et l'unité secondaire était l'animal. L'étude concernait tous les élevages de bovins se trouvant dans la zone et la répartition des exploitations enquêtées s'est faite selon la localisation et l'accessibilité des élevages (Tableau 1).

Dans la zone de transhumance du département du Lom et Djerem, l'étude s'est déroulée en saison sèche, période pendant laquelle les animaux vont en transhumance d'une région ou d'un pays à un autre. Les élevages échantillonnés étaient ceux choisis par les services vétérinaires en raison de l'accès facile aux parcs, la sécurité et une bonne connaissance de la maladie par les éleveurs et vendeurs disposés à répondre à nos préoccupations (Tableau 2).

\section{Collecte des données}

Au marché à bétail de Garoua-Boulai, une enquête prospective a été effectuée et l'unité épidémiologique était constituée du troupeau des bovins. Les animaux étaient regroupés selon leur appartenance et leur provenance. Les éleveurs et bergers de la zone savent bien reconnaître la fièvre aphteuse même s'ils ont tendance à sous-estimer la maladie. En compagnie des vétérinaires, ils nous montraient dans les troupeaux, les animaux suspects, en fonction des signes cliniques caractéristiques de la maladie (vésicules rompues, plaies dans la bouche, la langue, les tétines, la bande coronaire et l'espace inter digité, la boiterie, l'hyper salivation, les sécrétions provenant des naseaux et de la bouche).

Dans la zone de transhumance, une première enquête rétrospective nous a permis de recueillir les cas cliniques de FA observés et enregistrés pendant les 12 derniers mois (2017 à 2018) dans les rapports de la Délégation Départementale de l'Elevage et des Pêches du Lom et Djerem. Une deuxième enquête prospective a été effectuée par l'examen 
clinique des bovins suspects de FA. La méthodologie de Mannan et al. (2009) puis celle de Sarker et al. (2011) qui consiste à évaluer la prévalence de la FA par le diagnostic clinique a été appliquée lors de cette étude.

\section{Analyse statistique des données}

Les données obtenues ont été saisies dans le logiciel Excel ${ }^{\circledR}$ 2016. L'analyse statistique a été réalisée à l'aide du logiciel
Stata SE 14.2* (Stata Corp, 2015). Une régression logistique a été utilisée à l'échelle de la Délégation Départementale de l'Elevage et des Pêches du Lom et Djerem en prenant en compte comme variable explicative, le nombre de suspicions cliniques rapportées entre 2017 et 2018. La fonction "fweight" de Stata a été utilisée pour tenir compte du nombre d'observations. Une valeur de $\mathrm{p}<0,05$ a été considérée comme significative.

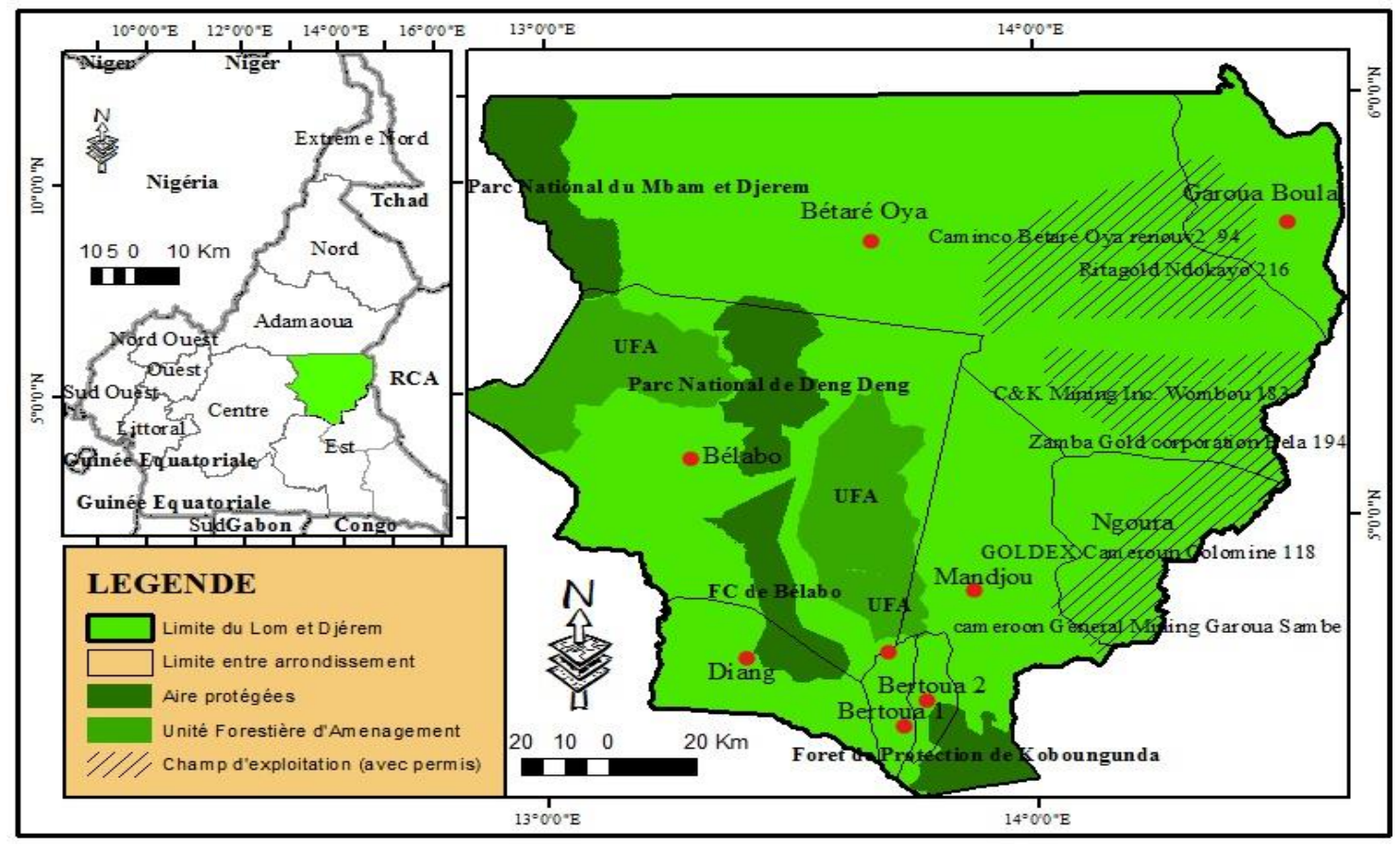

Figure 1: Localisation de la zone d'étude.

Tableau 1 : Répartition des bovins enquêtés dans le marché à bétail de Garoua-Boulai.

\begin{tabular}{lcccccc}
\hline \multicolumn{1}{c}{ Bovins } & \multicolumn{2}{c}{ Sexe } & \multicolumn{5}{c}{ Tranches d'âge } \\
\hline Race & Mâles & Femelles & $0-4$ ans & $4-6$ ans & $6-8$ ans & $>8$ ans \\
Bororo & 155 & 401 & 71 & 241 & 141 & 103 \\
Goudali & 16 & 42 & 11 & 19 & 15 & 13 \\
\hline \multicolumn{1}{c}{ Total } & 171 & 443 & 82 & 260 & 156 & 116 \\
\hline
\end{tabular}

Tableau 2 : Répartition des bovins atteints cliniquement de FA dans la zone de transhumance.

\begin{tabular}{lcccc}
\hline Arrondissements & Bovins positifs & Bovins négatifs & Prévalence (\%) & I \\
\hline Bétaré-Oya & 856 & 2066 & 29,29 & $27,64: 30,95$ \\
Garoua-Boulai & 430 & 1395 & 23,56 & $21,61: 25,51$ \\
Ngoura & 1008 & 2812 & 26,38 & $13,12: 35,15$ \\
\hline Total & 2294 & 6273 & 26,77 & \\
\hline
\end{tabular}

I= Intervalle de confiance. 
RESULTATS

\section{Prévalence de la fièvre aphteuse dans le marché à bétail de Garoua-Boulai}

Sur les 614 animaux suspects et issus de divers troupeaux de bovins, 58 ont présentés des signes cliniques caractéristiques de la FA, soit un taux de prévalence de 9,44\% (Tableau $3)$.

L'analyse de ce tableau montre que la prévalence de la FA est significativement ( $\mathrm{p}<$ $0,05)$ plus élevée chez les zébus goudali $(13,08 \%)$ que celle des zébus bororo $(5,46 \%)$. Les facteurs intrinsèques tels le sexe n'a pas eu d'influence sur la prévalence.

Les animaux ont été classés en quatre tranches d'âge selon le critère de classification de la dentition (Mannan et al., 2009) et la prévalence de la FA est présentée dans le Tableau 4.

Il ressort de ce tableau que la prévalence de la FA était plus élevée chez les animaux de la tranche d'âge de 0 à 4 ans. Une différence significative $(\mathrm{p}<0,05)$ a été observée entre les animaux de 0 à 4 ans et ceux de plus de 4 ans. Par contre, on n'enregistre pas une différence significative $(\mathrm{p}>0,05)$ entre les animaux de 4 à 6 ans et ceux âgés de plus de 8 ans.

\section{Prévalence et facteurs de risque de la fièvre aphteuse dans la zone de transhumance du département du Lom et Djerem}

Dans la zone de transhumance, les données recueillies auprès des registres des services vétérinaires sur la période allant de janvier 2017 à décembre 2018, font un état de 69 troupeaux d'un total de 8567 bovins suspects et de 2294 bovins ont présenté des signes cliniques caractéristiques de la FA soit un taux de prévalence de $26,77 \%$ (Tableau 5)

Il ressort de ce tableau qu'il n'existe pas de différence significative $(p>0,05)$ entre la prévalence de la fièvre aphteuse dans les différentes localités.

\section{Facteurs de risque de la diffusion de la fièvre aphteuse \\ > Identification des zones à risque}

Le marché à bétail de Garoua-Boulai et la zone de transhumance du Lom et Djerem dans la région de l'Est du Cameroun sont des zones à forte concentration d'animaux en raison de nombreux points d'eau, des zones de pâturage, de grands marchés à bétail, etc. Elles se répartissent à l'intérieur du département dans les arrondissements de Bétaré-Oya, Garoua-Boulai et Ngoura.

\section{> Mouvements des animaux et marchés à bétail}

Les mouvements perpétuels et incontrôlés des animaux (transhumance, commerce, nomadisme) constituent la cause essentielle de la diffusion des foyers de FA. A cela, il est nécessaire d'ajouter l'inefficacité des postes frontières de surveillance, l'absence de quarantaine des animaux, l'importation et l'exportation d'animaux vivants (sur pied) par les pays voisins et la notion de " maladie mineure" partagée par les éleveurs, la porosité des frontières, l'insécurité, la cohabitation avec la faune sauvage, la faible collaboration entre les pays de la sous-région Afrique centrale, etc. Ces mouvements d'animaux sont accompagnés de déplacements de personnes 'propriétaires, bouviers, marchands de bétail, négociants). Ils ont aussi lieu lors d'évènements sociaux tels que les mariages, les funérailles, les baptêmes, etc.

\section{> Sources d'infection}

Certaines zones sont reconnues comme réservoir du virus et considérées comme source d'infection primaire. Ces foyers sont à l'origine d'épizooties qui se propagent occasionnellement à d'autres régions qui sont considérées comme des sources d'infection secondaires. Tel est le cas en 2017 où 6 foyers confirmés de FA ont été enregistrés dans 5 départements de 3 régions du Cameroun (Tableau 6).

D'après nos observations, les zones d'infection secondaires et les zones à risque sont entretenues par les zones d'infection primaires. L'ensemble de ces zones forme un vaste territoire d'enzootie de FA maintenue principalement par la densité d'animaux sensibles, les mouvements d'animaux, les mouvements des personnes et secondairement par les produits animaux. La source d'infection dans le Lom et Djerem serait donc uniquement domestique et ne ferait pas intervenir la faune sauvage. 
Tableau 3: Prévalence de la FA au marché à bétail de Garoua-Boulai.

\begin{tabular}{lccc}
\hline \multicolumn{1}{c}{ Race } & Animaux suspects & Animaux positifs & Prévalence (\%) \\
\hline Bororo & 293 & 16 & 5,46 \\
Goudali & 321 & 42 & 13,08 \\
\hline Total & 614 & 58 & 9,44 \\
\hline
\end{tabular}

Tableau 4 : Prévalence de la FA en fonction de la tranche d'âge.

\begin{tabular}{ccccc}
\hline $\begin{array}{c}\text { Tranche } \\
\text { d'âge }\end{array}$ & Animaux examinés & $\begin{array}{c}\text { Animaux } \\
\text { positifs }\end{array}$ & Prévalence (\%) & $\begin{array}{c}\text { Intervalle de } \\
\text { confiance (\%) }\end{array}$ \\
\hline $0-4$ ans & 82 & 11 & 13,41 & {$[6,03: 20,79]$} \\
$4-6$ ans & 260 & 19 & 7,30 & {$[4,14: 10,47]$} \\
$6-8$ ans & 156 & 15 & 9,61 & {$[4,98: 14,24]$} \\
$>8$ ans & 116 & 13 & 11,20 & {$[5,46: 16,94]$} \\
\hline Total & 614 & 58 & 9,44 & {$[7,13: 11,76]$} \\
\hline
\end{tabular}

Tableau 5 : Prévalence de la FA dans la zone de transhumance du Lom et Djerem.

\begin{tabular}{lcccc}
\hline Arrondissements & $\begin{array}{c}\text { Animaux } \\
\text { suspects }\end{array}$ & $\begin{array}{c}\text { Cas cliniques } \\
\text { positifs }\end{array}$ & Prévalence (\%) & $\begin{array}{c}\text { Intervalle de } \\
\text { confiance (\%) }\end{array}$ \\
\hline Bétaré-Oya & 2922 & 856 & 29,29 & {$[27,64: 30,95]$} \\
Garoua-Boulai & 1825 & 430 & 23,56 & {$[21,61: 25,50]$} \\
Ngoura & 3820 & 1008 & 26,38 & {$[13,12: 35,15]$} \\
\hline Total & 8567 & 2294 & 26,77 & {$[25,85: 27,73]$} \\
\hline
\end{tabular}

Tableau 6 : Répartition des foyers de FA au Cameroun en 2017.

\begin{tabular}{lcccc}
\hline $\begin{array}{l}\text { Région } \\
\text { /département }\end{array}$ & $\begin{array}{c}\text { Nombre de } \\
\text { foyers }\end{array}$ & $\begin{array}{c}\text { Nombre de cas } \\
\text { suspects }\end{array}$ & $\begin{array}{c}\text { Nombre de cas } \\
\text { positifs }\end{array}$ & $\begin{array}{c}\text { Nombre de } \\
\text { morts }\end{array}$ \\
\hline - Adamaoua & 1 & 13 & 1 & 0 \\
$\quad$ Vina & 1 & 13 & 1 & 0 \\
- Extrême-Nord & 1 & 47 & 47 & 4 \\
Mayo-Tsanaga & 1 & 47 & 47 & 4 \\
- Nord-Ouest & 4 & 129 & 38 & 0 \\
Bui & 1 & 16 & 4 & 0 \\
Kumbo & 2 & 37 & 21 & 0 \\
Mezam & 1 & 76 & 13 & 0 \\
\hline Total & 6 & 189 & 86 & 4 \\
\hline
\end{tabular}




\section{DISCUSSION}

La présente étude a été menée pour déterminer la prévalence et les facteurs de risques de transmission de la FA au marché à bétail de Garoua-Boulai et dans la zone de transhumance du département du Lom et Djerem à l'Est du Cameroun.

La prévalence de la $\mathrm{FA}$ au marché à bétail de Garoua-boulai était de 9,44\%. Ce résultat corrobore celui de Kuate (2016) qui était de $11,36 \%$ dans une étude menée au grand Nord du Cameroun.

L'âge des bovins a eu une influence sur la prévalence de la FA. En effet, les jeunes animaux étaient significativement $(p<0,05)$ plus atteints que les adultes. Nos résultats concordent ceux de Miguel et al. (2013) sur la transmission de la FA bovine en Afrique. Ceci peut s'expliquer par le fait que les jeunes sont plus sensibles à la maladie et la mortalité est élevée que les adultes. Les zébus adultes, du fait de la pression des sélections ancestrales ne peuvent montrer que des manifestations sous cliniques ou douces (UA-BIRA, 2014). Il n'y a pas eu de différence significative $(p>0,05)$ entre le sexe. Malgré l'existence de barrières sanitaires, les animaux suspects de FA passent d'une localité à l'autre et échappent au contrôle. Le non isolement des animaux qui passent la barrière sanitaire constitue un facteur de risque pour toutes les espèces d'animaux réceptifs au virus aphteux.

La prévalence clinique de la FA dans la zone de transhumance du département du Lom et Djerem était de $26,77 \%$. Ce résultat est similaire à la prévalence de $24,51 \%$ rapportée par Mannan et al. (2009) au Bengladesh. Cette prévalence est supérieure à celle de Kuate (2016) qui était de $11,36 \%$ et à celle du marché à bétail de Garoua-Boulai qui était de 9,44\% . Elle est par contre inférieure à celle de Sorwar et al. (2016) qui était de 32,53\% chez les bovins au Bengladesh. La différence entre ces prévalences serait due à la méthode d'échantillonnage. Kuate (2016) a échantillonné sur les troupeaux de bovins suspects alors que Mannan et al. (2009) ont étudié sur les bovins venus en consultation dermatologique dans une clinique vétérinaire. Elles peuvent également provenir de la différence d'expression du virus en fonction du climat. Les cas de FA dans le marché à bétail et dans la zone de transhumance ont été enregistrés pendant la moitié de la saison pluvieuse, ce qui laisse suggérer l'influence des précipitations sur l'explosion des foyers de la FA. Ceci est en accord avec les observations de Megersa et al. (2009), de Florian et al. (2018) qui ont montré que les précipitations supérieures à $80 \mathrm{ml} / \mathrm{an}$ et les températures audessus de $24{ }^{\circ} \mathrm{C}$ ont une influence sur la diffusion du virus aphteux. Cela est contraire à celles de Couacy et al. (2006) qui ont observé des périodes de forte recrudescence en saison sèche et froide (décembre à février), sur les lieux de rassemblement tels que les points d'eau en saison des pluies (juin à septembre).

\section{Conclusion}

La présente étude a été menée pour déterminer la prévalence et les facteurs de risques de transmission de la FA qui expliquent son endémicité dans le marché à bétail de Garoua-Boulai et dans la zone de transhumance du département du Lom et Djerem dans la région de l'Est du Cameroun. L'épidémiologie de la FA et son impact économique dans la région de l'Est sont encore peu connus. La distribution et les facteurs de persistance du virus ne sont pas les mêmes dans toutes les zones et leurs connaissances pourraient aider à mieux comprendre l'épidémiologie de la maladie. Les principaux facteurs de risque de propagation de la maladie étaient : le non-respect des bonnes pratiques d'élevage (biosécurité), la transhumance des ruminants domestiques (à la recherche des pâturages et des points d'eau), les marchés à bétail, les zones de pâture et les points d'eau, la porosité des frontières, l'insécurité, l'exportation des animaux sur pied, la faible collaboration entre les pays de la sous-région de l'Afrique centrale. Aussi, une étude visant à mieux connaître la situation actuelle de la FA en déterminant la prévalence et en analysant les facteurs de risque qui peuvent influencer l'endémicité dans ces zones permettra d'estimer les effets de la FA sur les revenus des agriculteurs et de mieux identifier les options de maîtrise. Cette étude serait également de nature à aider à la prise de décision. Les foyers de FA signalés sur le terrain devraient être 
également vérifiés avant leur prise en compte effective afin d'éviter des déclarations à l'OIE qui ne reflètent pas la réalité sur le terrain.

\section{CONFLIT D'INTERETS} d'intérêts.

Les auteurs ne déclarent aucun conflit

\section{CONTRIBUTIONS DES AUTEURS}

Tous les auteurs ont contribué à la prise en charge des matériels didactique, technique et à la rédaction du manuscrit. Tous les auteurs ont lu et approuvé la version finale du manuscrit.

\section{REMERCIEMENTS}

Les auteurs expriment leurs sincères remerciements aux vétérinaires, éleveurs et vendeurs de bétail du département du Lom et Djerem, pour leur aide précieuse sur le terrain. Nos remerciements s'adressent particulièrement à M. Souyoudi Aboubakar, Délégué Départemental de l'Elevage, des Pêches et des Industries Animales du Lom et Djerem pour son accompagnement durant tout ce travail.

\section{REFERENCES}

Couacy-Hymann E, Aplogan GL, Sangaré O, Valarcher JF. 2006. Étude rétrospective de la Fièvre Aphteuse en Afrique de l'Ouest de 1970 à 2003. Rev. Sci. Tech. Off. Int. Epiz., 25(3):1013-1024.

FAO. 2015. Stratégic plan for the control of foot-and-mouth disease in Cameroon. FAO, 67p.

Florian D, Bronsvoort M, Samantha L. 2018. Circulation of foot-and-mouth disease virus in Africa and identification of the underlying constraints using phylogeographic methods. Bio. Rxiv. preprint., $21 \mathrm{p} . \quad$ DOI: https://doi.org/10.1101/358044

Grubman MJ, Baxt B. 2004. Foot-and-mouth disease. Clin. Microbiol. Rev., 17(2): 465493. DOI : $10.1128 / \mathrm{cmr} .17 .2 .465$ 493.2004

Kuate W. 2016. Prévalence et facteurs de risque signalés par les éleveurs de la Fièvre Aphteuse chez les bovins dans les départements de la Vina, du Diamaré et du Logone et Chari au Cameroun. Thèse de Méd.Vét., Ngaoundéré, 156p.

Mannan MA, Siddique MP, Uddin MZ, Parvez MM. 2009. Prevalence of foot and mouth disease (FMD) in cattle at Meghna Upazila in Comilla in Bangladesh. $J$. Bangladesh Agri. Univ., 7(2): 317-319. DOI: https://doi.org/10.3329/jbau.v7i2.4741

Megersa B, Beyene B, Abunna F, Regassa A. 2009. Risk factors for foot and mouth disease seroprevalence in indigenous cattle in Southern Ethiopia: The effect of production system. Trop Anim Health Prod., 41(6): 891-898. DOI: https://doi.org/10.1007/s11250-0089276-5

Miguel EV, Grosbois A, Caron T, GarineWichatitsky. 2013. Contacts and foot and mouth disease transmission from wild to domestic bovines in Africa. Ecosphere, 4(4): 51-59. DOI: https://doi.org/10.1890/ES12-00239-1

Orsel K, Bouma A, Dekker A, Stegeman JA. 2008. Foot and mouth disease virus transmission during the incubation period of the disease in piglets, lambs, calves and dairy cow. Preventive Veterinary Medicine, $\quad \mathbf{8 8}(2)$ : 158-163. DOI: https://doi.org/10.1016/j.prevetmed.2008 .09 .001

Sarker S, Talukder S, Haque MH, Islam MH, Gupta SD. 2011. Epidemiological study on foot-and-mouth disease in cattle: prevalence and risk factor assessment in Rajshahi, Bangladesh. Wayamba Journal of Animal Science, (1299745268): 71-73.

Sorwar MG, Rahman MA, Hasan MN, Islam MS. 2016. Prevalence of foot and mouth disease at Chuadanga Sadar Upazilla in Bangladesh. Bangl. J. Vet. Med., 14(1): 43-49. DOI: https://doi.org/10.3329/bjvm.v14i1.2882 21

UA-BIRA. 2014. Méthodes et Procédures Standard (MPS) pour le Contrôle de la fièvre aphteuse dans la Grande Corne de l'Afrique. UA-BIRA, Nairobi, 38p. 